COMMUNICATIONS IN

ANALYSIS AND GEOMETRY

Volume 11, Number 5, 837-858, 2003

\title{
Prescribing a Higher Order Conformal Invariant on $S^{n}$
}

\author{
Simon BRendle
}

\section{Introduction.}

An important problem in differential geometry is to construct conformal metrics on $S^{2}$ whose Gauss curvature equals a given positive function $f$. This problem is equivalent to finding a solution of the equation

$$
-\Delta_{0} w=f e^{2 w}-1,
$$

where $\Delta_{0}$ denotes the Laplace operator associated with the standard metric $g_{0}$ on $S^{2}$. J. Moser [22] proved that this equation has a solution if the function $f$ satisfies the condition $f(x)=f(-x)$ for all $x \in S^{2}$. The general case was studied by S.-Y. A. Chang, M. Gursky, and P. Yang [10, 11, 14].

A. Bahri and J. M. Coron [4, 5] and R. Schoen and D. Zhang [24] constructed metrics with prescribed scalar curvature on $S^{3}$. J. F. Escobar and R. Schoen [18] studied the prescribed scalar curvature problem on manifolds which are not necessarily conformally equivalent to the standard sphere.

Our aim is to generalize these results to higher dimensions. Let $g$ be a conformal metric on $S^{4}$. We denote by $R$ the scalar curvature of $g$ and by Ric the Ricci tensor of $g$. Moreover, we denote by $\Delta$ the Laplace operator with respect to the metric $g$. A natural conformal invariant in dimension four is

$$
Q=-\frac{1}{6}\left(\Delta R-R^{2}+3|R i c|^{2}\right)
$$

The quantity $Q$ plays an important role in conformal geometry, see $[7,12,15]$. Indeed, the quantity $Q$ enjoys similar properties as the Gauss curvature in dimension two. For a given positive function $f$ on $S^{4}$, we want to construct a conformal metric $g$ on $S^{4}$ such that

$$
Q=6 f .
$$

If we denote by $g_{0}$ the standard metric on $S^{4}$, then this problem is equivalent to the equation

$$
\Delta_{0}^{2} w-2 \Delta_{0} w=6\left(f e^{4 w}-1\right) .
$$


If the function $f$ satisfies the condition $f(x)=f(-x)$ for all $x \in S^{4}$, then this equation has a solution. The solution can be constructed by means of an evolution equation, see [9].

More generally, we can consider the standard sphere $S^{n}$, where $n$ is even. By the work of C. Fefferman and R. Graham [19, 20], there exists a conformally invariant self-adjoint operator with leading term $\left(-\Delta_{0}\right)^{\frac{n}{2}}$. On the standard sphere $S^{n}$, this operator is given by

$$
P_{0}=\prod_{k=1}^{\frac{n}{2}}\left(-\Delta_{0}+(k-1)(n-k)\right),
$$

see $[8,12]$. We consider the equation

$$
P_{0} w=(n-1) !\left(f e^{n w}-1\right)
$$

for some positive function $f$ on $S^{n}$. This is a semilinear elliptic equation of order $n$ involving the critical Sobolev exponent. We assume that the function $f$ satisfies the non-degeneracy condition

$$
\nabla_{0} f(p) \quad \Longrightarrow \quad \Delta_{0} f(p) \neq 0 .
$$

Moreover, we identify the group of conformal transformations on $S^{n}$ with the unit ball in $\mathbb{R}^{n+1}$. Moreover, we consider the map

$$
H: B^{n+1} \rightarrow \mathbb{R}^{n+1}, \quad \sigma \mapsto\left(\int_{S^{n}} f \circ \sigma x_{i} d V_{0}\right)_{1 \leq i \leq n+1}
$$

Then we have the following result:

Theorem 1.1. Suppose that $f$ satisfies the non-degeneracy condition and

$$
\operatorname{deg}(H, 0) \neq 0
$$

Then the equation

$$
P_{0} w=(n-1) !\left(f e^{n w}-1\right)
$$

has a solution.

As a consequence, we obtain:

Corollary 1.2. Suppose that $f$ satisfies the non-degeneracy condition and

$$
\sum_{\nabla_{0} f(p)=0, \Delta_{0} f(p)<0}(-1)^{\operatorname{ind}(f, p)} \neq 1 .
$$


Then the equation

$$
P_{0} w=(n-1) !\left(f e^{n w}-1\right)
$$

has a solution.

An index criterion similar to that in Corollary 1.2 was introduced by A. Bahri and J. M. Coron [5] in their work on the prescribed scalar curvature problem. Related results were established by Z. Djadli, A. Malchiodi and M. Ahmedou [16, 17].

In Section 2, we consider solutions of the equation

$$
P_{0} w=(n-1) !\left(f e^{n w}-1\right)
$$

satisfying the normalization condition

$$
\int_{S^{n}} e^{n w} x_{j} d V_{0}=0
$$

for $1 \leq j \leq n+1$. Using the estimates for the Paneitz operator from [12], we can show that the function $w$ is bounded in $H^{n}$. If the function $f$ is close to 1 , we establish an estimate of the form

$$
\|w\|_{H^{n}} \leq C\|f-1\|_{L^{2}}
$$

for some constant $C$.

In Section 3, we show that the a-priori estimates remain valid even if the normalization condition is dropped. The proof relies on the Kazdan-Warner identity (see [12]) and the non-degeneracy condition for $f$.

In Section 4, we use a topological degree argument to show that the equation

$$
P_{0} w=(n-1) !\left(f e^{n w}-1\right)
$$

has a solution.

\section{A-priori estimates for solutions satisfying a normalization condition.}

Let $f$ be a positive function on $S^{n}$, and let $w$ be a function which satisfies the equation

$$
P_{0} w=(n-1) !\left(f e^{n w}-1\right)
$$

and the normalization condition

$$
\int_{S^{n}} e^{n w} x_{j} d V_{0}=0
$$

for $1 \leq j \leq n+1$. We begin with a simple estimate. 
Lemma 2.1. The function $w$ satisfies

$$
\|w-\bar{w}\|_{W}{ }_{\frac{n}{2}, p} \leq C
$$

for all $p<2$.

Proof. The function $P_{0} w$ satisfies

$$
\left|P_{0} w\right| \leq P_{0} w+2(n-1) !
$$

hence

$$
f_{S^{n}}\left|P_{0} w\right| d V_{0} \leq 2(n-1) !
$$

Using Green's formula, we obtain

$$
\|w-\bar{w}\|_{W^{\frac{n}{2}, p}} \leq C
$$

for all $p<2$.

Using the normalization condition, it is possible to derive an improved Sobolev inequality for the function $w$. The proof follows ideas of T. Aubin $[2,3]$ and is included here for completeness.

Proposition 2.2. The function $w$ satisfies the inequality

$$
\log \left(\int_{S^{n}} e^{n(w-\bar{w})} d V_{0}\right) \leq f_{S^{n}} \frac{n}{4(n-1) !} w P_{0} w d V_{0}+C .
$$

Proof. We use the inequality

$$
\begin{aligned}
\log \left(\int_{S^{n}} e^{n u} d V_{0}\right) & \leq f_{S^{n}} \frac{n}{2(n-1) !}\left(\left(-\Delta_{0}\right)^{\frac{n}{4}} u\right)^{2} d V_{0} \\
& +f_{S^{n}} n u d V_{0}+C
\end{aligned}
$$

(see $[1,6,12])$. Without loss of generality, we may assume that

$$
\int_{S^{n}} e^{n w} d V_{0} \leq C \int_{\left\{x_{n+1} \geq 2 \delta\right\}} e^{n w} d V_{0}
$$

We first consider the case

$$
\int_{\left\{x_{n+1} \geq \delta\right\}}\left(\left(-\Delta_{0}\right)^{\frac{n}{4}} w\right)^{2} d V_{0} \leq \int_{\left\{x_{n+1} \leq \delta\right\}}\left(\left(-\Delta_{0}\right)^{\frac{n}{4}} w\right)^{2} d V_{0} .
$$


This implies

$$
\int_{\left\{x_{n+1} \geq \delta\right\}}\left(\left(-\Delta_{0}\right)^{\frac{n}{4}} w\right)^{2} d V_{0} \leq \int_{S^{n}} \frac{1}{2}\left(\left(-\Delta_{0}\right)^{\frac{n}{4}} w\right)^{2} d V_{0} .
$$

We choose a cut-off function $\eta$ such that $\eta=1$ for $x_{n+1} \geq 2 \delta$ and $\eta=0$ for $x_{n+1} \leq \delta$. For $u=\eta(w-\bar{w})$ we obtain

$$
\begin{aligned}
\log \left(\int_{S^{n}} e^{n \eta(w-\bar{w})} d V_{0}\right) & \leq f_{S^{n}} \frac{n}{2(n-1) !}\left(\left(-\Delta_{0}\right)^{\frac{n}{4}}(\eta(w-\bar{w}))\right)^{2} d V_{0} \\
& +f_{S^{n}} n \eta(w-\bar{w}) d V_{0}+C
\end{aligned}
$$

From this it follows that

$$
\log \left(\int_{\left\{x_{n+1} \geq 2 \delta\right\}} e^{n(w-\bar{w})} d V_{0}\right) \leq f_{S^{n}} \frac{n}{2(n-1) !} \eta^{2}\left(\left(-\Delta_{0}\right)^{\frac{n}{4}} w\right)^{2} d V_{0}+C .
$$

Therefore, we obtain

$$
\log \left(\int_{S^{n}} e^{n(w-\bar{w})} d V_{0}\right) \leq \int_{S^{n}} \frac{n}{4(n-1) !}\left(\left(-\Delta_{0}\right)^{\frac{n}{4}} w\right)^{2} d V_{0}+C
$$

We now consider the case

$$
\int_{\left\{x_{n+1} \leq \delta\right\}}\left(\left(-\Delta_{0}\right)^{\frac{n}{4}} w\right)^{2} d V_{0} \leq \int_{\left\{x_{n+1} \geq \delta\right\}}\left(\left(-\Delta_{0}\right)^{\frac{n}{4}} w\right)^{2} d V_{0}
$$

This implies

$$
\int_{\left\{x_{n+1} \leq \delta\right\}}\left(\left(-\Delta_{0}\right)^{\frac{n}{4}} w\right)^{2} d V_{0} \leq \int_{S^{n}} \frac{1}{2}\left(\left(-\Delta_{0}\right)^{\frac{n}{4}} w\right)^{2} d V_{0} .
$$

We choose a cut-off function $\eta$ such that $\eta=1$ for $x_{n+1} \leq 0$ and $\eta=0$ for $x_{n+1} \geq \delta$. For $u=\eta(w-\bar{w})$ we obtain

$$
\begin{aligned}
\log \left(\int_{S^{n}} e^{n \eta(w-\bar{w})} d V_{0}\right) & \leq f_{S^{n}} \frac{n}{2(n-1) !}\left(\left(-\Delta_{0}\right)^{\frac{n}{4}}(\eta(w-\bar{w}))\right)^{2} d V_{0} \\
& +f_{S^{n}} n \eta(w-\bar{w}) d V_{0}+C
\end{aligned}
$$

From this it follows that

$$
\log \left(\int_{\left\{x_{n+1} \leq 0\right\}} e^{n(w-\bar{w})} d V_{0}\right) \leq f_{S^{n}} \frac{n}{2(n-1) !} \eta^{2}\left(\left(-\Delta_{0}\right)^{\frac{n}{4}} w\right)^{2} d V_{0}+C
$$


Using the inequality

$$
\begin{aligned}
\int_{S^{n}} e^{n(w-\bar{w})} d V_{0} & \leq C \int_{\left\{x_{n+1} \geq 2 \delta\right\}} e^{n(w-\bar{w})} d V_{0} \\
& \leq C \int_{\left\{x_{n+1} \geq 2 \delta\right\}} e^{n(w-\bar{w})} x_{n+1} d V_{0} \\
& =-C \int_{\left\{x_{n+1} \leq 2 \delta\right\}} e^{n(w-\bar{w})} x_{n+1} d V_{0} \\
& \leq-C \int_{\left\{x_{n+1} \leq 0\right\}} e^{n(w-\bar{w})} x_{n+1} d V_{0} \\
& \leq C \int_{\left\{x_{n+1} \leq 0\right\}} e^{n(w-\bar{w})} d V_{0},
\end{aligned}
$$

we obtain

$$
\log \left(\int_{S^{n}} e^{n(w-\bar{w})} d V_{0}\right) \leq \int_{S^{n}} \frac{n}{4(n-1) !}\left(\left(-\Delta_{0}\right)^{\frac{n}{4}} w\right)^{2} d V_{0}+C .
$$

This proves the assertion.

On the other hand, S.-Y. A. Chang and P. Yang [12] established the following estimate:

Proposition 2.3. Assume that

$$
0<m \leq f \leq M
$$

Then the function $w$ satisfies

$$
f_{S^{n}} \frac{n}{2(n-1) !} w P_{0} w d V_{0}-\log \left(\int_{S^{n}} e^{n(w-\bar{w})} d V_{0}\right) \leq C .
$$

Combining these statements, we obtain:

Corollary 2.4. Assume that

$$
0<m \leq f \leq M
$$

Then the function $w$ satisfies

$$
\int_{S^{n}} w P_{0} w d V_{0} \leq C
$$

As a consequence, we obtain: 
Proposition 2.5. If the function $f$ satisfies

$$
0<m \leq f \leq M
$$

then the function $w$ satisfies the estimate $\|w\|_{H^{n}} \leq C$.

Proof. It follows from Corollary 2.4 that

$$
\|w-\bar{w}\|_{H^{\frac{n}{2}}} \leq C .
$$

Using an inequality of N. Trudinger, we obtain

$$
\int_{S^{n}} e^{n(w-\bar{w})} d V_{0} \leq C
$$

Since

$$
P_{0} w=(n-1) !\left(f e^{n w}-1\right)
$$

we obtain

$$
f_{S^{n}} f e^{n w} d V_{0}=1
$$

This implies

$$
\frac{1}{M} \leq f_{S^{n}} e^{n w} d V_{0} \leq \frac{1}{m}
$$

From this it follows that

$$
-C \leq \bar{w} \leq C .
$$

Thus, we conclude that $\|w\|_{H^{\frac{n}{2}}} \leq C$, hence

$$
\int_{S^{n}} e^{2 n w} d V_{0} \leq C
$$

by Trudinger's inequality. From this it follows that

$$
\int_{S^{n}}\left(P_{0} w\right)^{2} d V_{0} \leq C
$$

Since $\bar{w}$ is bounded, the assertion follows.

In the remaining part of this section, we assume that the function $f$ is close to 1 .

Lemma 2.6. For every $\varepsilon>0$, there exists a real number $\delta>0$ with the following property: If the function $f$ satisfies

$$
0<m \leq f \leq M
$$

and

$$
\|f-1\|_{L^{2}} \leq \delta
$$

then the function $w$ satisfies the estimate $\|w\|_{H^{n}} \leq \varepsilon$. 
Proof. We consider a sequence of functions $w_{k}$ satisfying

$$
P_{0} w_{k}=(n-1) !\left(f_{k} e^{n w_{k}}-1\right)
$$

and

$$
\int_{S^{n}} e^{n w_{k}} x_{j} d V_{0}=0
$$

for $1 \leq j \leq n+1$. We assume that

$$
0<m \leq f_{k} \leq M
$$

and

$$
\left\|f_{k}-1\right\|_{L^{2}} \rightarrow 0
$$

By Proposition 2.5, the function satisfies the estimate $\left\|w_{k}\right\|_{H^{n}} \leq C$. Hence, by passing to a subsequence, we may assume that

$$
\left\|w_{k}-w\right\|_{L^{\infty}} \rightarrow 0
$$

for some function $w$. Then the function $w$ satisfies

$$
P_{0} w=(n-1) !\left(e^{n w}-1\right) .
$$

From this it follows that $w$ is smooth. Moreover, it follows from the results in [13] that the metric $e^{2 w} g_{0}$ agrees with the standard metric $g_{0}$ up to conformal transformations. Using the normalization condition

$$
\int_{S^{n}} e^{n w} x_{j} d V_{0}=0
$$

for $1 \leq j \leq n+1$, we conclude that $w=0$. This implies

$$
\left\|w_{k}\right\|_{L^{\infty}} \rightarrow 0
$$

Since

$$
P_{0} w_{k}=(n-1) !\left(f_{k} e^{n w_{k}}-1\right)
$$

it follows that

$$
\left\|P_{0} w_{k}\right\|_{L^{2}} \rightarrow 0
$$

Therefore, we obtain

$$
\left\|w_{k}\right\|_{H^{n}} \rightarrow 0
$$

This proves the assertion. 
Proposition 2.7. Assume that the function $f$ satisfies

$$
0<m \leq f \leq M
$$

and

$$
\|f-1\|_{L^{2}} \leq \delta
$$

Then the function $w$ satisfies an estimate of the form

$$
\|w\|_{H^{n}} \leq C\|f-1\|_{L^{2}}
$$

Proof. The function $w$ satisfies

$$
P_{0} w-n ! w=(n-1) !(f-1) e^{n w}+(n-1) !\left(e^{n w}-n w-1\right)
$$

and

$$
\int_{S^{n}} w x_{j} d V_{0}=-\int_{S^{n}} \frac{1}{n}\left(e^{n w}-n w-1\right) x_{j} d V_{0}
$$

for $1 \leq j \leq n+1$. Using the Sobolev embedding theorem, we obtain

$$
\|w\|_{L^{\infty}} \leq C\|w\|_{H^{n}} \leq C \varepsilon .
$$

From this it follows that

$$
\left\|P_{0} w-n ! w\right\|_{L^{2}} \leq C\|f-1\|_{L^{2}}+C \varepsilon\|w\|_{L^{2}}
$$

and

$$
\left|\int_{S^{n}} w x_{j} d V_{0}\right| \leq C \varepsilon\|w\|_{L^{2}}
$$

for $1 \leq j \leq n+1$. Thus, we conclude that

$$
\|w\|_{H^{n}} \leq C\|f-1\|_{L^{2}}+C \varepsilon\|w\|_{L^{2}},
$$

hence

$$
\|w\|_{H^{n}} \leq C\|f-1\|_{L^{2}}
$$

This proves the assertion.

Proposition 2.8. Let $f$ be a function with

$$
\|f-1\|_{L^{2}} \leq \delta
$$

Then there exists a unique pair $(w, \Lambda) \in H^{n} \times \mathbb{R}^{n+1}$ such that

$$
P_{0} w=(n-1) !\left(\left(f-\sum_{j=1}^{n+1} \Lambda_{j} x_{j}\right) e^{n w}-1\right)
$$


and

$$
\int_{S^{n}} e^{n w} x_{j} d V_{0}=0
$$

for $1 \leq j \leq n+1$ and $\|(w, \Lambda)\|_{H^{n} \times \mathbb{R}^{n+1}} \leq \varepsilon$.

Proof. Let

$$
\mathcal{S}=\left\{w \in H^{n}: \int_{S^{n}} e^{n w} x_{j} d V_{0}=0 \quad \text { for } 1 \leq j \leq n+1\right\} .
$$

We define a map

$$
\Phi: \mathcal{S} \times \mathbb{R}^{n+1} \rightarrow L^{2}
$$

by

$$
\Phi(w, \Lambda)=e^{-n w} P_{0} w+(n-1) ! e^{-n w}+\sum_{j=1}^{n+1}(n-1) ! \Lambda_{j} x_{j} .
$$

We denote by

$$
\Phi^{\prime}: T \mathcal{S} \times \mathbb{R}^{n+1} \rightarrow L^{2}
$$

the differential of $\Phi$ at the point $(0,0)$. We have

$$
T \mathcal{S}=\left\{w \in H^{n}: \int_{S^{n}} w x_{j} d V_{0}=0 \quad \text { for } 1 \leq j \leq n+1\right\}
$$

and

$$
\Phi^{\prime}(w, \Lambda)=P_{0} w-n ! w+\sum_{j=1}^{n+1}(n-1) ! \Lambda_{j} x_{j} .
$$

Therefore, the map $\Phi^{\prime}$ is bijective. The implicite function theorem implies that $\Phi$ is a bijective map from a neighbourhood of $(0,0)$ in $\mathcal{S} \times \mathbb{R}^{n+1}$ to a neighbourhood of $(n-1)$ ! in $L^{2}$. Since

$$
\|f-1\|_{L^{2}} \leq \delta
$$

there exists a pair $(w, \Lambda) \in \mathcal{S} \times \mathbb{R}^{n+1}$ such that

$$
\Phi(w, \Lambda)=(n-1) ! f
$$

and

$$
\|(w, \Lambda)\|_{H^{n} \times \mathbb{R}^{n+1}} \leq \varepsilon .
$$

From this the assertion follows. 


\section{A-priori estimates for solutions in the absence of a} normalization condition.

Let $f$ be a fixed positive function on $S^{n}$. In this section, we establish the following result:

Proposition 3.1. Let $w$ be a solution of the equation

$$
P_{0} w=(n-1) !\left((s f+1-s) e^{n w}-1\right)
$$

for some $0<s \leq 1$. Then the function $w$ satisfies the estimate $\|w\|_{H^{n}} \leq C$.

Proof. Assume that there exists a sequence of functions $w_{k}$ satisfying

$$
P_{0} w_{k}=(n-1) !\left(\left(s_{k} f+1-s_{k}\right) e^{n w_{k}}-1\right)
$$

for some $0<s_{k} \leq 1$ and

$$
\left\|w_{k}\right\|_{H^{n}} \rightarrow \infty
$$

We choose conformal transformations $\sigma_{k}$ such that

$$
\int_{S^{n}} e^{n \tilde{w}_{k}} x_{j} d V_{0}=0
$$

for $1 \leq j \leq n+1$, where

$$
e^{2 \tilde{w}_{k}} g_{0}=\sigma_{k}^{*}\left(e^{2 w_{k}} g_{0}\right) \text {. }
$$

Then the functions $\tilde{w}_{k}$ satisfy the equation

$$
P_{0} \tilde{w}_{k}=(n-1) !\left(\left(s_{k} f+1-s_{k}\right) \circ \sigma_{k} e^{n \tilde{w}_{k}}-1\right) .
$$

Since $f$ is a fixed positive function on $S^{n}$, we have

$$
0<m \leq\left(s_{k} f+1-s_{k}\right) \circ \sigma_{k} \leq M .
$$

Hence, it follows from Proposition 2.5 that $\left\|\tilde{w}_{k}\right\|_{H^{n}} \leq C$. Since

$$
\left\|w_{k}\right\|_{H^{n}} \rightarrow \infty
$$

we conclude that the sequence $\sigma_{k}$ tends to infinity. This implies

$$
\left\|\left(s_{k} f+1-s_{k}\right) \circ \sigma_{k}-e^{-n r_{k}}\right\|_{L^{2}}=o(1)
$$

for a suitable constant $r_{k}$. Using Lemma 2.6, we obtain

$$
\left\|\tilde{w}_{k}-r_{k}\right\|_{H^{n}}=o(1) .
$$


Moreover, the Kazdan-Warner identity (see [12]) implies that

$$
\int_{S^{n}}\left\langle d\left(f \circ \sigma_{k}\right), d x_{j}\right\rangle e^{n\left(\tilde{w}_{k}-r\right)} d V_{0}=0
$$

for $1 \leq j \leq n+1$. If we identify $S^{n}$ with $\mathbb{R}^{n} \cup\{\infty\}$ via the stereographic projection, then we may assume that the conformal transformation $\sigma_{k}$ is given by

$$
\sigma_{k}(y)=\frac{1}{t_{k}} y
$$

for a suitable sequence $t_{k} \rightarrow \infty$. The pull-back of the standard metric on $S^{n}$ under the stereographic projection is given by

$$
\left(g_{0}\right)_{i j}=\frac{4}{\left(1+|y|^{2}\right)^{2}} \delta_{i j}
$$

Moreover, we have

$$
x_{j}=\frac{2 y_{j}}{1+|y|^{2}}
$$

for $1 \leq j \leq n$ and

$$
x_{n+1}=-\frac{1-|y|^{2}}{1+|y|^{2}} .
$$

This implies

$$
d x_{j}=\frac{2}{1+|y|^{2}} d y_{j}-\sum_{i=1}^{n} \frac{4 y_{i} y_{j}}{\left(1+|y|^{2}\right)^{2}} d y_{i}
$$

for $1 \leq j \leq n$ and

$$
d x_{n+1}=\sum_{i=1}^{n} \frac{4 y_{i}}{\left(1+|y|^{2}\right)^{2}} d y_{i}
$$

Using the formula

$$
f(y)=f(0)+\sum_{i=1}^{n} \alpha_{i} y_{i}+\frac{1}{2} \sum_{i, j=1}^{n} \beta_{i j} y_{i} y_{j}+o\left(|y|^{2}\right),
$$

we obtain

$$
\left(f \circ \sigma_{k}\right)(y)=f(0)+\frac{1}{t_{k}} \sum_{i=1}^{n} \alpha_{i} y_{i}+\frac{1}{2 t_{k}^{2}} \sum_{i, j=1}^{n} \beta_{i j} y_{i} y_{j}+o\left(\frac{|y|^{2}}{t_{k}^{2}}\right),
$$

hence

$$
d\left(f \circ \sigma_{k}\right)(y)=\frac{1}{t_{k}} \sum_{i=1}^{n} \alpha_{i} d y_{i}+\frac{1}{t_{k}^{2}} \sum_{i, j=1}^{n} \beta_{i j} y_{j} d y_{i}+o\left(\frac{|y|}{t_{k}^{2}}\right) .
$$


From this it follows that

$$
\begin{aligned}
0 & =\int_{S^{n}}\left\langle d\left(f \circ \sigma_{k}\right), d x_{j}\right\rangle e^{n\left(\tilde{w}_{k}-r_{k}\right)} d V_{0} \\
& =\int_{S^{n}}\left\langle d\left(f \circ \sigma_{k}\right), d x_{j}\right\rangle d V_{0}+o\left(\frac{1}{t_{k}}\right) \\
& =\frac{1}{t_{k}} \int_{\mathbb{R}^{n}} \frac{2^{n-1} \alpha_{j}}{\left(1+|y|^{2}\right)^{n-1}} d y_{1} \cdots d y_{n}-\frac{1}{t_{k}} \sum_{i=1}^{n} \int_{\mathbb{R}^{n}} \frac{2^{n} \alpha_{i} y_{i} y_{j}}{\left(1+|y|^{2}\right)^{n}} d y_{1} \cdots d y_{n}+o\left(\frac{1}{t_{k}}\right) \\
& =\frac{1}{t_{k}} \int_{\mathbb{R}^{n}} \frac{2^{n-1} \alpha_{j}}{\left(1+|y|^{2}\right)^{n-1}} d y_{1} \cdots d y_{n}-\frac{1}{t_{k}} \int_{\mathbb{R}^{n}} \frac{2^{n} \alpha_{j} y_{j}^{2}}{\left(1+|y|^{2}\right)^{n}} d y_{1} \cdots d y_{n}+o\left(\frac{1}{t_{k}}\right) \\
& =\frac{1}{t_{k}} \int_{\mathbb{R}^{n}} \frac{2^{n-1} \alpha_{j}}{\left(1+|y|^{2}\right)^{n-1}} d y_{1} \cdots d y_{n}-\frac{1}{t_{k}} \int_{\mathbb{R}^{n}} \frac{2^{n} \alpha_{j}|y|^{2}}{n\left(1+|y|^{2}\right)^{n}} d y_{1} \cdots d y_{n}+o\left(\frac{1}{t_{k}}\right) \\
& =\frac{1}{t_{k}} \int_{\mathbb{R}^{n}} \frac{2^{n-1} \alpha_{j}\left(n+(n-2)|y|^{2}\right)}{n\left(1+|y|^{2}\right)^{n}} d y_{1} \cdots d y_{n}+o\left(\frac{1}{t_{k}}\right)
\end{aligned}
$$

for $1 \leq j \leq n$. Thus, we conclude that $\alpha_{j}=o(1)$ for $1 \leq j \leq n$. From this it follows that

$$
\left\|\left(s_{k} f+1-s_{k}\right) \circ \sigma_{k}-e^{-n r_{k}}\right\|_{L^{2}} \leq o\left(\frac{1}{t_{k}}\right)
$$

where $e^{-n r_{k}}=s_{k} f(0)+1-s_{k}$. This implies

$$
\left\|\tilde{w}_{k}-r_{k}\right\|_{H^{n}} \leq o\left(\frac{1}{t_{k}}\right)
$$

Using this estimate, we obtain

$$
\begin{aligned}
0 & =\int_{S^{n}}\left\langle d\left(f \circ \sigma_{k}\right), d x_{n+1}\right\rangle e^{n\left(\tilde{w}_{k}-r_{k}\right)} d V_{0} \\
& =\int_{S^{n}}\left\langle d\left(f \circ \sigma_{k}\right), d x_{n+1}\right\rangle d V_{0}+o\left(\frac{1}{t_{k}^{2}}\right) \\
& =\frac{1}{t_{k}^{2}} \sum_{i, j=1}^{n} \int_{\mathbb{R}^{n}} \frac{2^{n} \beta_{i j} y_{i} y_{j}}{\left(1+|y|^{2}\right)^{n}} d y_{1} \cdots d y_{n}+o\left(\frac{1}{t_{k}^{2}}\right) \\
& =\frac{1}{t_{k}^{2}} \sum_{i=1}^{n} \int_{\mathbb{R}^{4}} \frac{2^{n} \beta_{i i}|y|^{2}}{n\left(1+|y|^{2}\right)^{n}} d y_{1} \cdots d y_{n}+o\left(\frac{1}{t_{k}^{2}}\right) .
\end{aligned}
$$

Thus, we conclude that

$$
\sum_{i=1}^{n} \beta_{i i}=0
$$


Therefore, the concentration point $p$ satisfies

$$
\nabla_{0} f(p)=0
$$

and

$$
\Delta_{0} f(p)=0 .
$$

This contradicts the non-degeneracy condition.

\section{Existence results.}

Let

$$
\mathcal{M}_{s}=\left\{w \in H^{n}: f_{S^{n}}(s f+1-s) e^{n w} d V_{0}=1\right\} .
$$

We define a map

$$
\Psi_{s}: \mathcal{M}_{s} \rightarrow H^{n}
$$

by

$$
\Psi_{s}(w)=w-(n-1) ! P_{0}^{-1}\left((s f+1-s) e^{n w}-1\right) .
$$

We first show that the degree of $\Psi_{s}$ is independent of $s$.

Proposition 4.1. We have

$$
\operatorname{deg}\left(\Psi_{1}, 0\right)=\operatorname{deg}\left(\Psi_{s}, 0\right)
$$

for all $0<s \leq 1$

Proof. It follows from Proposition 3.1 that the set

$$
\left\{(s, w): 0<s \leq 1, w \in \mathcal{M}_{s} \text { and } \Psi_{s}(w)=0\right\}
$$

is bounded in $\mathbb{R} \times H^{n}$. The assertion is now a consequence of the homotopy invariance of the degree (see [23]).

We now choose $0<s \leq 1$ sufficiently small. By Proposition 2.8, for every conformal transformation $\sigma$, there exists a unique function $\tilde{w}_{\sigma}$ which satisfies

$$
P_{0} \tilde{w}_{\sigma}=(n-1) !\left(\left((s f+1-s) \circ \sigma-\sum_{j=1}^{n+1} \Lambda_{\sigma, j} x_{j}\right) e^{n \tilde{w}_{\sigma}}-1\right)
$$

and

$$
\int_{S^{n}} e^{n \tilde{w}_{\sigma}} x_{j} d V_{0}=0
$$


for $1 \leq j \leq n+1$ and

$$
\left\|\left(\tilde{w}_{\sigma}, \Lambda_{\sigma}\right)\right\|_{H^{n} \times \mathbb{R}^{n+1}} \leq \varepsilon .
$$

Using Proposition 2.7, we obtain $\left\|\tilde{w}_{\sigma}\right\|_{H^{n}} \leq C s$. We now define functions $w_{\sigma}$ by

$$
e^{2 \tilde{w}_{\sigma}} g_{0}=\sigma^{*}\left(e^{2 w_{\sigma}} g_{0}\right) .
$$

Then the function $w_{\sigma}$ satisfies the equation

$$
P_{0} w_{\sigma}=(n-1) !\left(\left((s f+1-s)-\sum_{j=1}^{n+1} \Lambda_{\sigma, j} x_{j} \circ \sigma^{-1}\right) e^{n w_{\sigma}}-1\right) .
$$

In the first step, we show that the zeroes of $\Psi_{s}$ are in one-to-one correspondence with the zeroes of $\Lambda$.

Proposition 4.2. A function $w$ satisfies $\Psi_{s}(w)=0$ if and only if there exists a conformal transformation $\sigma$ such that $w=w_{\sigma}$ and $\Lambda_{\sigma}=0$.

Proof. Suppose that $w \in \mathcal{M}_{s}$ satisfies $\Psi_{s}(w)=0$. Then the function $w$ satisfies the equation

$$
P_{0} w=(n-1) !\left((s f+1-s) e^{n w}-1\right) .
$$

We choose a conformal transformation $\sigma$ such that

$$
\int_{S^{n}} e^{n \tilde{w}} x_{j} d V_{0}=0
$$

for $1 \leq j \leq n+1$, where

$$
e^{2 \tilde{w}} g_{0}=\sigma^{*}\left(e^{2 w} g_{0}\right)
$$

Then the function $\tilde{w}$ satisfies the equation

$$
P_{0} \tilde{w}=(n-1) !\left((s f+1-s) \circ \sigma e^{n \tilde{w}}-1\right) .
$$

If $s$ is sufficiently small, then we have

$$
\|(s f-s) \circ \sigma\|_{L^{2}} \leq \delta .
$$

Using Proposition 2.6, we obtain

$$
\|\tilde{w}\|_{H^{n}} \leq \varepsilon .
$$


Hence, it follows from the uniqueness statement in Proposition 2.8 that $\tilde{w}=\tilde{w}_{\sigma}$ and $\Lambda_{\sigma}=0$. Conversely, if $\sigma$ is a conformal transformation satisfying $\Lambda_{\sigma}=0$, then the function $w_{\sigma}$ belongs to the space $\mathcal{M}_{s}$ and $\Psi_{s}\left(w_{\sigma}\right)=0$.

Let $\sigma$ be a conformal transformation satisfying $\Lambda_{\sigma}=0$, and let $\Lambda^{\prime}$ be the differential of $\Lambda$ at $\sigma$. Furthermore, we denote by $\Psi_{s}^{\prime}$ the differential of $\Psi_{s}$ at $w_{\sigma}$. We want to compare the number of negative eigenvalues of $\Lambda^{\prime}$ and $\Psi_{s}^{\prime}$.

To this end, we differentiate the identity

$$
P_{0} w_{\tau}=(n-1) !\left(\left((s f+1-s)-\sum_{j=1}^{n+1} \Lambda_{\tau, j} x_{j} \circ \tau^{-1}\right) e^{n w_{\tau}}-1\right)
$$

with respect to $\tau$. This gives a collection of functions $u_{i}$ such that

$$
P_{0} u_{i}=n !(s f+1-s) e^{n w_{\sigma}} u_{i}-(n-1) ! \sum_{j=1}^{n+1} \Lambda_{i, j}^{\prime} x_{j} \circ \sigma^{-1} e^{n w_{\sigma}}
$$

for $1 \leq i \leq n+1$. By definition of $w_{\sigma}$, we have

$$
\int_{S^{n}} e^{n w_{\sigma}} x_{j} \circ \sigma^{-1} d V_{0}=\int_{S^{n}} e^{n \tilde{w}_{\sigma}} x_{j} d V_{0}=0
$$

for $1 \leq j \leq n+1$. Let $v_{j}$ be the solution of the linear equation

$$
P_{0} v_{j}=-x_{j} \circ \sigma^{-1} e^{n w_{\sigma}} .
$$

Then we obtain the identity

$$
P_{0} u_{i}=n !(s f+1-s) e^{n w_{\sigma}} u_{i}+(n-1) ! \sum_{j=1}^{n+1} \Lambda_{i, j}^{\prime} x_{j} \circ \sigma^{-1} e^{n w_{\sigma}} .
$$

Thus, we conclude that $u_{i} \in T \mathcal{M}_{s}$ and

$$
\Psi_{s}^{\prime}\left(u_{i}\right)=(n-1) ! \sum_{j=1}^{n+1} \Lambda_{i, j}^{\prime} v_{j}
$$

We now establish precise estimates for the functions $u_{i}$ and $v_{j}$.

Lemma 4.3. The function $u_{i}$ satisfies the estimate

$$
\left\|u_{i}+x_{i} \circ \sigma^{-1}\right\|_{H^{n}} \leq C s .
$$


Proof. Since

$$
\|(s f+1-s) \circ \tau-(s f+1-s) \circ \sigma\|_{L^{2}} \leq C s \operatorname{dist}(\tau, \sigma),
$$

it follows from the proof of Proposition 2.8 that

$$
\left\|\tilde{w}_{\tau}-\tilde{w}_{\sigma}\right\|_{H^{n}} \leq C s \operatorname{dist}(\tau, \sigma) .
$$

This implies

$$
\left\|\tilde{w}_{\tau} \circ \tau^{-1}-\tilde{w}_{\sigma} \circ \sigma^{-1}\right\|_{H^{n}} \leq C s \operatorname{dist}(\tau, \sigma) .
$$

Using the relations

$$
\tilde{w}_{\sigma} \circ \sigma^{-1}=w_{\sigma}+\frac{1}{n} \log \operatorname{det} d \sigma \circ \sigma^{-1}
$$

and

$$
\tilde{w}_{\tau} \circ \tau^{-1}=w_{\tau}+\frac{1}{n} \log \operatorname{det} d \tau \circ \tau^{-1}
$$

we obtain

$$
\left\|w_{\tau}-w_{\sigma}+\frac{1}{n} \log \operatorname{det} d \tau \circ \tau^{-1}-\frac{1}{n} \log \operatorname{det} d \sigma \circ \sigma^{-1}\right\|_{H^{n}} \leq C s \operatorname{dist}(\tau, \sigma),
$$

hence

$$
\left\|w_{\tau}-w_{\sigma}-\frac{1}{n} \log \operatorname{det} d\left(\tau^{-1} \circ \sigma\right) \circ \sigma^{-1}\right\|_{H^{n}} \leq C s \operatorname{dist}(\tau, \sigma) .
$$

From this it follows that

$$
\left\|u_{i}+x_{i} \circ \sigma^{-1}\right\|_{H^{n}} \leq C s .
$$

This proves the assertion.

Lemma 4.4. The function $v_{j}$ satisfies the estimate

$$
\left\|n ! v_{j}+x_{j} \circ \sigma^{-1}\right\|_{H^{n}} \leq C s .
$$

Proof. Since $-\Delta_{0} x_{j}=n x_{j}$, we obtain

$$
P_{0} x_{j}=\prod_{k=1}^{\frac{n}{2}}(n+(k-1)(n-k)) x_{j}=\prod_{k=1}^{\frac{n}{2}} k(n-k+1) x_{j}=n ! x_{j} .
$$

Using the conformal invariance of the Paneitz operator, we conclude that

$$
P_{0}\left(x_{j} \circ \sigma^{-1}\right) \operatorname{det} d \sigma \circ \sigma^{-1}=n ! x_{j} \circ \sigma^{-1},
$$


hence

$$
P_{0}\left(x_{j} \circ \sigma^{-1}\right) e^{n \tilde{w}_{\sigma} \circ \sigma^{-1}}=n ! x_{j} \circ \sigma^{-1} e^{n w_{\sigma}} .
$$

This implies

$$
n ! P_{0} v_{j}=-P_{0}\left(x_{j} \circ \sigma^{-1}\right) e^{n \tilde{w}_{\sigma} \circ \sigma^{-1}} .
$$

Using the estimate

$$
\left\|\tilde{w}_{\sigma}\right\|_{H^{n}} \leq C s
$$

we obtain

$$
\left\|n ! v_{j}+x_{j} \circ \sigma^{-1}\right\|_{H^{n}} \leq C\left\|P_{0}\left(n ! v_{j}+x_{j} \circ \sigma^{-1}\right)\right\|_{L^{2}} \leq C s .
$$

This proves the assertion.

Proposition 4.5. If $s$ is sufficiently small, then the degree of $\Psi_{s}$ coincides with the degree of $\Lambda$.

Proof. By Lemma 4.3 and Lemma 4.4, the finite-dimensional approximations of $\Psi_{s}^{\prime}$ are of the form

$$
\left(\begin{array}{cc}
\Lambda^{\prime} E & \Lambda^{\prime} F \\
X & Y
\end{array}\right)^{T}
$$

where

$$
\|E-1\| \leq C s
$$

and

$$
\|F\| \leq C s
$$

Using the identity

$$
\left(\begin{array}{cc}
\Lambda^{\prime} E & \Lambda^{\prime} F \\
X & Y
\end{array}\right)=\left(\begin{array}{cc}
\Lambda^{\prime} & 0 \\
X E^{-1} & Y-X E^{-1} F
\end{array}\right)\left(\begin{array}{cc}
E & F \\
0 & 1
\end{array}\right)
$$

we obtain

$$
\begin{aligned}
\operatorname{det}\left(\begin{array}{cc}
\Lambda^{\prime} E & \Lambda^{\prime} F \\
X & Y
\end{array}\right) & =\operatorname{det}\left(\begin{array}{cc}
\Lambda^{\prime} & 0 \\
X E^{-1} & Y-X E^{-1} F
\end{array}\right) \operatorname{det}\left(\begin{array}{cc}
E & F \\
0 & 1
\end{array}\right) \\
& =\operatorname{det} \Lambda^{\prime} \operatorname{det}\left(Y-X E^{-1} F\right) \operatorname{det} E
\end{aligned}
$$

Hence, if $s$ is sufficiently small, then $\operatorname{det}\left(\begin{array}{cc}\Lambda^{\prime} E & \Lambda^{\prime} F \\ X & Y\end{array}\right)$ and $\operatorname{det} \Lambda^{\prime}$ have the same sign. Thus, we conclude that

$$
\operatorname{deg}\left(\Psi_{s}, 0\right)=\operatorname{deg}(\Lambda, 0)
$$


if $s$ is sufficiently small.

We now identify the the group of conformal transformations on $S^{n}$ with the unit ball in $\mathbb{R}^{n+1}$. We consider the map

$$
H: B^{n+1} \rightarrow \mathbb{R}^{n+1}, \quad \sigma \mapsto\left(\int_{S^{n}} f \circ \sigma x_{i} d V_{0}\right)_{1 \leq i \leq n+1}
$$

Then we have the following result:

Proposition 4.6. If $s$ is sufficiently small, then the degree of $\Lambda$ coincides with the degree of $H$.

Proof. Using the Kazdan-Warner identity, we obtain

$$
\int_{S^{n}}\left\langle d((s f+1-s) \circ \sigma)-\sum_{j=1}^{n+1} \Lambda_{\sigma, j} d x_{j}, d x_{i}\right\rangle e^{n \tilde{w}_{\sigma}} d V_{0}=0 .
$$

This implies

$$
s \int_{S^{n}}\left\langle d(f \circ \sigma), d x_{i}\right\rangle e^{n \tilde{w}_{\sigma}} d V_{0}=\sum_{j=1}^{n+1} \Lambda_{\sigma, j} \int_{S^{n}}\left\langle d x_{j}, d x_{i}\right\rangle e^{n \tilde{w}_{\sigma}} d V_{0} .
$$

Therefore, the degree of $\Lambda$ coincides with the degree of the map

$$
G: B^{n+1} \rightarrow \mathbb{R}^{n+1}, \quad \sigma \mapsto\left(\int_{S^{n}}\left\langle d(f \circ \sigma), d x_{i}\right\rangle e^{n \tilde{w}_{\sigma}} d V_{0}\right)_{1 \leq i \leq n+1}
$$

On the other hand,

$$
\begin{aligned}
|G(\sigma)-n H(\sigma)| & \leq \sum_{i=1}^{n+1}\left|\int_{S^{n}}\left\langle d(f \circ \sigma), d x_{i}\right\rangle e^{n \tilde{w}_{\sigma}} d V_{0}-n \int_{S^{n}} f \circ \sigma x_{i} d V_{0}\right| \\
& \leq \sum_{i=1}^{n+1}\left|\int_{S^{n}}\left\langle d(f \circ \sigma), d x_{i}\right\rangle\left(e^{n \tilde{w}_{\sigma}}-1\right) d V_{0}\right| \\
& \leq C s .
\end{aligned}
$$

If $s$ is sufficiently small, then $G$ and $H$ are homotopic, and therefore the degree of $G$ agrees with the degree of $H$.

Combining these statements, we obtain

$$
\operatorname{deg}\left(\Psi_{1}, 0\right)=\operatorname{deg}(H, 0) .
$$


By assumption, we have $\operatorname{deg}(H, 0) \neq 0$, hence $\operatorname{deg}\left(\Psi_{1}, 0\right) \neq 0$. Therefore, there exists a function $w \in \mathcal{M}_{1}$ such that $\Psi_{1}(w)=0$. This implies

$$
f_{S^{n}} f e^{n w} d V_{0}=1
$$

and

$$
w-(n-1) ! P_{0}^{-1}\left(f e^{n w}-1\right)=0 .
$$

Thus, we conclude that

$$
P_{0} w=(n-1) !\left(f e^{n w}-1\right) .
$$

\section{References.}

[1] D. Adams, A sharp inequality of J. Moser for higher order derivatives, Ann. of Math. 128, 385-398 (1988)

[2] T. Aubin, Meilleures constantes dans le théorème d'inclusion de Sobolev et un théorème de Fredholm non linéaire pour la transformation conforme de la courbure scalaire, J. Funct. Anal. 32, 148-174 (1979)

[3] T. Aubin, Nonlinear Analysis on Manifolds, Monge-Ampère Equations, Springer-Verlag, New York (1982)

[4] A. Bahri and J.M. Coron, On a nonlinear elliptic equation involving the critical Sobolev exponent: The effect of the topology of the domain, Comm. Pure Appl. Math. 41, 253-290 (1988)

[5] A. Bahri and J. M. Coron, The scalar curvature on the standard threedimensional sphere, J. Funct. Anal. 95, 106-172 (1991)

[6] W. Beckner, Sharp Sobolev inequalities on the sphere and the MoserTrudinger inequality, Ann. of Math. 138, 213-242 (1993)

[7] T. Branson, S.-Y. A. Chang and P. Yang, Estimates and extremal problems for the log-determinant on 4-manifolds, Comm. Math. Phys. 149, 241-262 (1992)

[8] T. Branson, Sharp inequalities, the functional determinant, and the complementary series, Trans. Amer. Math. Soc. 347, 3671-3742 (1995)

[9] S. Brendle, Global existence and convergence for a higher order flow in conformal geometry, to appear in Ann. of Math. 
[10] S.-Y. A. Chang and P. Yang, Prescribing Gauss curvature on $S^{2}$, Acta Math. 159, 215-259 (1987)

[11] S.-Y. A. Chang and P. Yang, A perturbation result in prescribing scalar curvature on $S^{n}$, Duke Math. J. 64, 27-69 (1991)

[12] S.-Y. A. Chang and P. Yang, Extremal metrics of zeta functional determinants on 4-manifolds, Ann. of Math. 142, 171-212 (1995)

[13] S.-Y. A. Chang and P. Yang, On uniqueness of solutions of $n$-th order differential equations in conformal geometry, Math. Res. Lett. 4, 91-102 (1997)

[14] S.-Y. A. Chang, M. Gursky and P. Yang, The scalar curvature on 2and 3-spheres, Comm. PDE 1, 205-229 (1993)

[15] S.-Y. A. Chang, M. Gursky and P. Yang, An Equation of Monge-Ampere type in conformal geometry and four-manifolds of positive Ricci curvature, to appear in Ann. of Math.

[16] Z. Djadli, A. Malchiodi and M. Ahmedou, Prescribing a fourth order conformal invariant on the standard sphere, Part I: a perturbation result, preprint (2000)

[17] Z. Djadli, A. Malchiodi and M. Ahmedou, Prescribing a fourth order conformal invariant on the standard sphere, Part II: blow-up analysis and applications, preprint (2000)

[18] J. F. Escobar and R. Schoen, Conformal metrics with prescribed scalar curvature, Invent. Math. 86, 243-254 (1986)

[19] C. Fefferman and R. Graham, Conformal invariants, Astérisque, 95-116 (1985)

[20] C. Fefferman and R. Graham, Q-curvature and Poincaré metrics, prepint (2001)

[21] C. S. Lin, A classification of solutions of a conformally invariant fourth order equation in $\mathbb{R}^{n}$, Comm. Math. Helv. 73, 206-231 (1998)

[22] J. Moser, On a non-linear problem in differential geometry, in Dynamical Systems, ed. M. Peixoto, Academic Press, New York (1973)

[23] L. Nirenberg, Topics in Nonlinear Functional Analysis, Lectures, Courant Institute, New-York (1974) 
[24] R. Schoen and D. Zhang, Prescribed scalar curvature on the $n$-sphere, Calc. Var. PDE 4, 1-25 (1996)

Received June 26, 2002. 